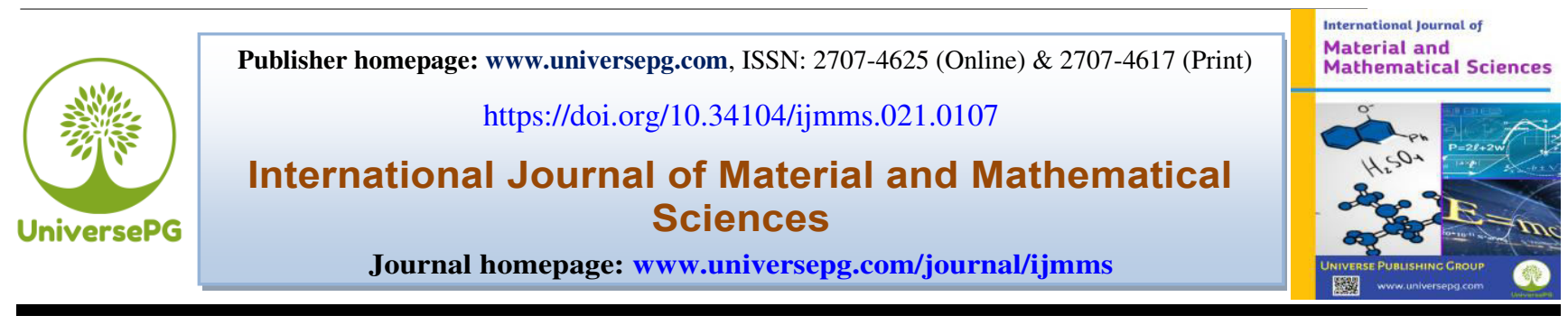

\title{
Cooperative Spectrum Sensing with Selection Diversity Reception in Cognitive Radio
}

\author{
Md. Farukuzzaman Khan ${ }^{1}$ and Mohammad Alamgir Hossain ${ }^{2} *$ \\ ${ }^{1 \& 2}$ Department of Computer Science and Engineering, Islamic University (IU), Jhenidah-Kushtia, Bangladesh. \\ *Correspondence: alamgir@cse.iu.ac.bd (Mohammad Alamgir Hossain, Assistant Professor, Department of Computer \\ Science and Engineering, IU, Jhenidah-Kushtia, Bangladesh).
}

\begin{abstract}
Spectrum sensing is the key component of cognitive radio technology. But, detection is compromised when a user reports shadowing or fading consequences. In such instances, the customer cannot apprehend between an unexploited band and a profound fade. Hence, communal spectrum sensing is suggested to optimize sensing overall performance. We recognition performance of communal Spectrum Sensing with Selection Diversity Reception in Cognitive Radio. This study presents a simulation evaluation of choice diversity Reception based totally on fusion rule. Fusion rule is finished at the fusion centre (FC) to make the very last selection about the presence of PU. This leads that spectrum sensing is enthusiastically in the presence of Rayleigh.
\end{abstract}

Keywords: Cognitive radio, Fusion rules, Cooperative spectrum sensing, Fading channels, and Energy detection.

\section{INTRODUCTION:}

The radio frequency (RF) spectrum is currently managed by government agencies under an exclusive usage scheme. Due to the explosive development of wireless applications, it is evident that the available frequencies cannot meet the increasing demand. Current investigations confirmed that there is present good sized underutilization of the allotted frequencies (FCC, 2002). The spectral under utilization can be resolved by means of permitting a secondary user to entree an authorized band whilst the primary consumer (PU) is absent. CR is appreciably agreed to be the maximum encouraging approach for assuaging RF spectral insufficiency. Present day investigations confirmed that there is present sizeable underutilization of the allotted frequencies (FCC, 2002). The spectral under utilization can be resolved via allowing a secondary person to entree a certified band whilst the primary user (PU) is absent. $\mathrm{CR}$ is notably agreed to be the maximum encouraging technique for assuaging RF Spectral efficiUniversePG I www.universepg.com ency. Energy detection (Urkowitz, 1967) is normally utilized in spectrum sensing, as it has a low execution issue and does now not want to channel kingdom records (CSI) (Sun et al., 2010). Because of the a few multipath fading, a cognitive radio may additionally fail to assertion the presence of the PU and then will entree the licensed channel and purpose interference to the PU. The act of communal spectrum sensing with disposing of cognitive radios in Rayleigh dwindle channel has been expected in (Nallagonda et al., 2011; Nallagonda et al., 2012; Alamgir et al., 2012; Alamgir et al., 2012a; Ahmed et al., 2012). It improves the detection performance. In previous work on cooperative spectrum sensing has shown that area diversity can growth the chance of detection (Chen et al., 2008; Chen et al., 2011; Wang et al., 2008; and Wang et al., 2009).

The utilization of most ratio combining (MRC), selection combining (SC), square law combining (SLC), and square law selection (SLS) intelligence 
fusion schemes (Alam and alam, 2020). Rayleigh wane channels became analysed in (Digham et al., 2007). Herath et al. measured the overall completion of MRC, SC, SLC, and SLS over more than one Nakagami-?? Fading channels in (Herath et al., 2009; Herath and Rajatheva, 2009), respectively. In this paper, we only studied selection combining (SC) data fusion scheme based in F. F. Digham by cooperating four number of $\mathrm{CR}$ users. The rest of this aticle is organized as follows. In segment 2, the system model is delivered. In phase 3 , detection and false alarm probability of non-fading AWGN and fading channel together with Rayleigh, Ricean and Nakagami are defined. Communal spectrum sensing over various fading channels is derived in segment 4 . The simulation result and dialogue are offered in segment 5. Subsequently, we draw our conclusions in phase 6.

\section{SYSTEM MODEL}

The local spectrum sensing is to arbitrate between the below two hypotheses,

$$
x(t)=\left\{\begin{array}{cc}
n(t) & H_{0} \\
h s(t)+n(t) & H_{1}
\end{array}\right.
$$

Where, $x(t)$ is the signal accepted by secondary operator and $s(t)$ is primary operator's transmitted signal, $n(t)$ is the supplement white Gaussian noise and $h$ is the magnitude gain of the channel. The energy calm in the frequency domain is standing for by $\mathrm{Y}$ which provides as a verdict statistic. Following the study of Urkowitz, $Y$ may be represented to have the following distribution,

$$
Y=\left\{\begin{array}{cc}
\chi^{2}{ }^{2 T W} & H_{0} \\
\chi^{2}{ }^{2 T W}(2 \gamma), & H_{1}
\end{array}\right.
$$

Where, $\chi^{2}{ }_{2 T W}$ and $\chi^{2}{ }_{2 T W}(2 \gamma)$ denote central and non-central chi-square placements respectively, each with $2 T W$ degrees of liberation and a non-centrality parameter of $2 \gamma$ for the latter placement. For simplicity we presume that time-bandwidth goods, $T W$, are an integer number which we designate by $u$.

\section{DETECTION AND FALSE ALARM PROB- ABILITIES}

In this phase, we provide the average detection chance over Rayleigh fading channels and in closed shape (Digham et al., 2003). In communications principle, Rayleigh distributions are used to model scattered a signal that attain a receiver with the aid of more than one path (Islam et al., 2020). In nonfading surroundings the common chance of false alarm, the common chance of detection, and the common opportunity of missed detection are given, respectively,

$$
\begin{aligned}
& P_{d}=P\left\{Y>\lambda \mid H_{1}\right\}=Q_{u}(\sqrt{2 \gamma}, \sqrt{\lambda}) \\
& P_{f}=P\left\{Y>\lambda \mid H_{0}\right\}=\frac{\Gamma(u, \lambda / 2)}{\Gamma(u)}
\end{aligned}
$$

and,

$$
P_{m}=1-P_{d}
$$

Where, $\lambda$ denotes the energy threshold. $\Gamma($.$) and$ $\Gamma(.,$.$) are complete and incomplete gamma functions$ respectively (Gradshteyn and Ryzhik, 2007) and $Q_{u}(.,$.$) is the generalized Marcum Q-function$ defined as follows,

$$
Q_{u}(a, b)=\int_{0}^{\infty} \frac{x^{u}}{a^{u-1}} e^{-\frac{x^{2}+a^{2}}{2}} I_{u-1}(a x) d x
$$

Where, $I_{u-1}($.$) is the modified Bessel function of$ $(u-1)$ th order If the signal strength is unknown, we will first set the false alarm probability to a selected constant. With the aid of equation (four), the detection threshold? May be decided. Then, for the fixed quantity of samples 2TW the detection possibility can be evaluated by way of substituting the? In (3). As expected, is unbiased of? Considering under there is no primary signal gift. While $h$ is various due to fading, equation (3) gives the chance of detection as a characteristic of the immediately SNR,

In this situation, the common chance of detection may be derived by means of averaging (three) over fading records (Lee et al., 2009), (6) where f? (x) is the opportunity distribution feature (PDF) of SNR beneath fading. In this case, the average possibility of detection can be derived through averaging (three) over fading statistics (Lee et al., 2009).

$$
P_{d}=\int_{x} Q_{u}(\sqrt{2 \gamma}, \sqrt{\lambda}) f_{\gamma}(x) d x
$$

Where, in $f(x)$ is the probability distribution characteristic (PDF) of SNR beneath fading. 


\section{A. Rayleigh fading channels}

When the received signal sign consists of a large quantity of aircraft waves, for some sorts of scattering environments, the received signal has a Rayleigh distribution (Stuber, 2002). If the sign amplitude follows a Rayleigh distribution, then the SNR? Follows an exponential PDF given by $f(\gamma)=\frac{1}{\gamma} \exp \left(-\frac{\gamma}{\gamma}\right), \gamma \geq 0$

In this example, a closed-shape formulation for $P_{d}$ may be obtained (after some manipulation) by substituting $f_{\gamma}(x)$ in (6),

$$
\begin{aligned}
& \bar{P}_{\text {dRay }}=e^{-\frac{\lambda}{2}} \sum_{k=0}^{u-2} \frac{1}{k !}\left(\frac{\lambda}{2}\right)^{k}+\left(\frac{1+\bar{\gamma}}{\bar{\gamma}}\right)^{u-1} \\
& \times\left(e^{-\frac{\lambda}{2(1+\bar{\gamma})}}-e^{-\frac{\lambda}{2} \sum_{k=0}^{u-2} \frac{1}{k}\left(\frac{\lambda \bar{\lambda}}{2(1+\bar{\gamma})}\right)}\right)
\end{aligned}
$$

\section{B. Selection Combining}

In the SC diversity scheme, the branch with maximum SNR, $\gamma_{\max }$, is to be selected. The PDF of $\gamma_{\max }$ for IID Rayleigh branches is known to be given by

$$
f_{\gamma_{\max }}(\gamma)=\frac{L}{\gamma}\left(1-e^{-\gamma / \bar{\gamma}}\right)^{L-1} e^{-\gamma / \bar{\gamma}}
$$

This PDF can be rewritten as

$$
f_{\gamma_{\max }}(\gamma)=L \sum_{i=0}^{L-1} \frac{(-1)^{i}}{i+1}\left(\begin{array}{c}
L-1 \\
i
\end{array}\right) \frac{1}{\bar{\gamma} /(i+1)} e^{-\frac{\gamma}{\gamma(i+1)}}
$$

The PDF in (29) represents a weighted sum of exponential varieties each with parameter $\frac{\bar{\gamma}}{i+1}$. Hence, the average $P_{d}$ for the SC diversity scheme, $\bar{P}_{d S C}$, can be evaluated as -

$$
\bar{P}_{d S C}=L \sum_{i=0}^{L-1} \frac{(-1)^{i}}{i+1}\left(\begin{array}{c}
L-1 \\
i
\end{array}\right) \bar{P}_{d R a y}\left(\frac{\bar{\gamma}}{i+1}\right)
$$

Where

$\bar{P}_{d R a y}\left(\frac{\bar{\gamma}}{i+1}\right)$ Is the $\bar{P}_{d R a y}$ obtained in (16) with the replacement of each $\bar{\gamma}$ by $\left(\frac{\bar{\gamma}}{i+1}\right)$.

\section{COOPERATIVE SPECTRUM SENSING OVER VARIOUS FADING CHANNELS}

With a difficult decision counting rule, the fusion center implements an $\mathrm{n}$ out-of-M rule that decides at the signal gift hypothesis each time at least $\mathrm{k}$ out of the N CR user selections indicate $H_{1}$. The probability of detection at the fusion center (Spyros et al., 2008) is given by

$$
P_{d}=\sum_{l=k}^{N}\left(\begin{array}{c}
N \\
l
\end{array}\right) P_{d, i}{ }^{l}\left(1-P_{d, i}\right)^{N-l}
$$

Where $P_{d, i}$ is the probability of detection for each individual CR user as defined by (3) and (6).

AND-rule: in this rule, if all the local selections dispatched to the decision maker are one, the final decision made by means of the decision maker is one. The fusion middle's decision is calculated through logic AND of the received tough decision records. Cooperative detection performance with this fusion rule may be evaluated by setting $\mathrm{ok}=\mathrm{N}$ in $k=N$ in eq. (9),

$$
P_{d, A N D}=P_{d, i}{ }^{N}
$$

OR-rule: in this rule, if someone of the local selections sent to the decision maker is a logical one, the final selection made through the decision maker is one. Cooperative detection overall performance with this fusion rule may be evaluated with the aid of setting $\mathrm{k}=1$ in eq. (9).

$$
P_{d, O R}=1-\left(1-P_{d, i}\right)^{N}
$$

MAJORITY-rule: in this rule, if half or greater of the neighborhood decisions dispatched to the selection maker are the final decision made with the aid of the selection maker is one.

Cooperative detection overall performance with this fusion rule may be evaluated via putting $k=\lfloor N / 2\rfloor$ in eq. (9).

$$
P_{d, M A J}=\sum_{l=[N / 2\rfloor}^{N}\left(\begin{array}{c}
N \\
l
\end{array}\right) P_{d, i}{ }^{l}\left(1-P_{d, i}\right)^{N-l}
$$

Where, $\lfloor$.$\rfloor represents the floor operator.$

\section{SIMULATIN RESULT AND DISCUSSION}

All simulation was done on MATLAB over three different fading under Rayleigh fading channel. We 
described the receiver through its complementary ROC curves for different values of probability of false alarm and Cognitive Radio user. Fig 1(a) Complementary ROC curves for L-branch SC reception ( $\mathrm{u}=5, \mathrm{~N}=4, \mathrm{SNR}=20, \mathrm{~L}=1,2,3$, 4) Fig $\mathbf{1}(\mathbf{b})$ Complementary ROC curves for L-branch SC reception $(\mathrm{u}=5, \mathrm{~N}=4, \mathrm{SNR}=20, \mathrm{~L}=1,2,3$, 4) Fig $\mathbf{1}(\mathbf{c})$ Complementary ROC curves for L-branch SC reception ( $\mathrm{u}=5, \mathrm{~N}=4, \mathrm{SNR}=20, \mathrm{~L}=1,2,3,4)$ Fig 1(a), Fig 1(b), and Fig 1(c) show complementary ROC curves of the 4 user's spectrum sensing in Rayleigh fading following AND rule, OR rule and MAJORITY rule respectively. Average SNR and $\mathrm{u}$ are assumed to be $20 \mathrm{~dB}$ and 5 respectively. We look at that the OR rule has the better performance than AND, and MAJORITY rule in various channels.

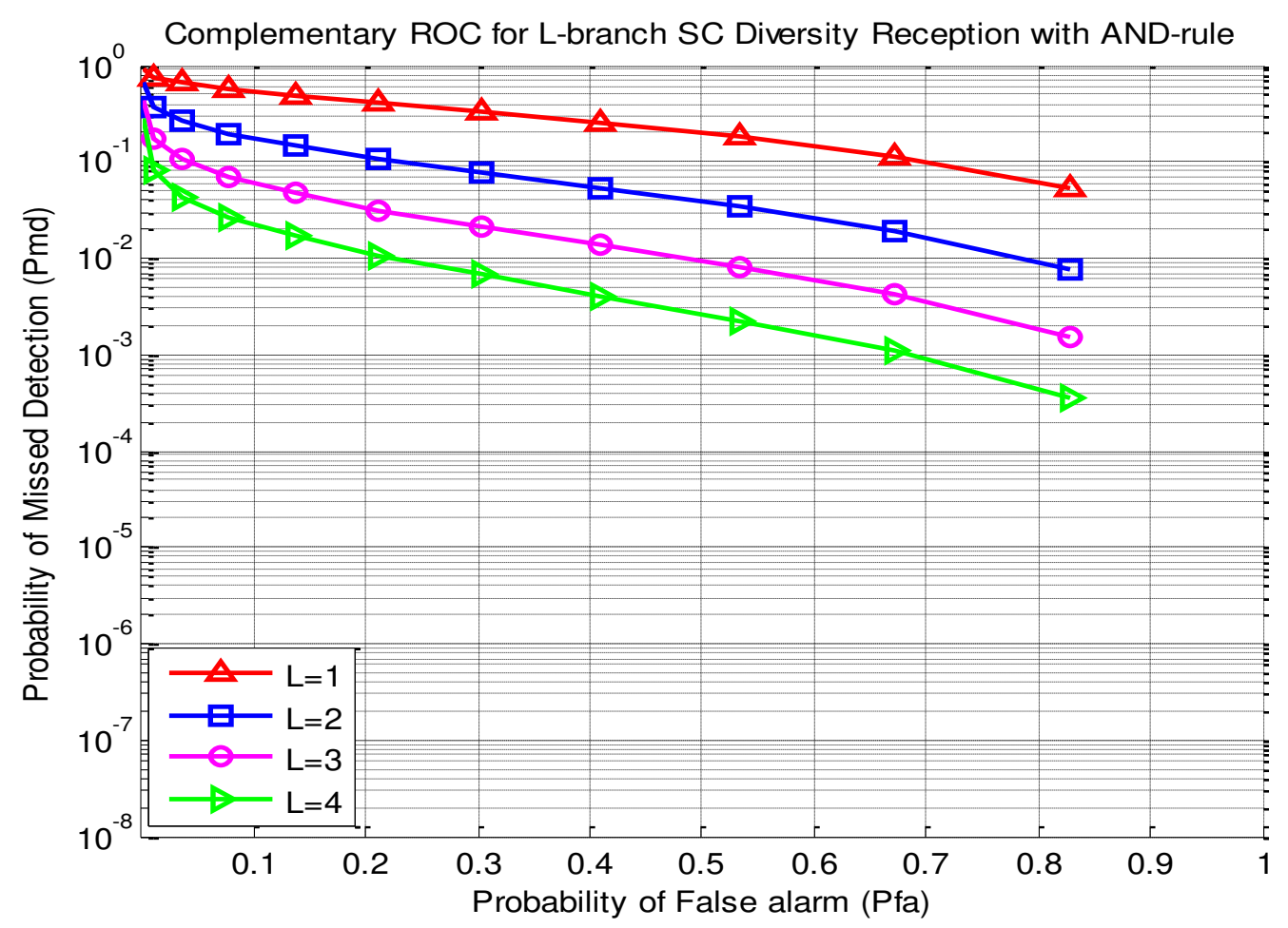

Fig 1(a): Complementary ROC curves for L-branch $S C$ reception $(u=5, N=4, S N R=20, L=1,2,3,4)$.

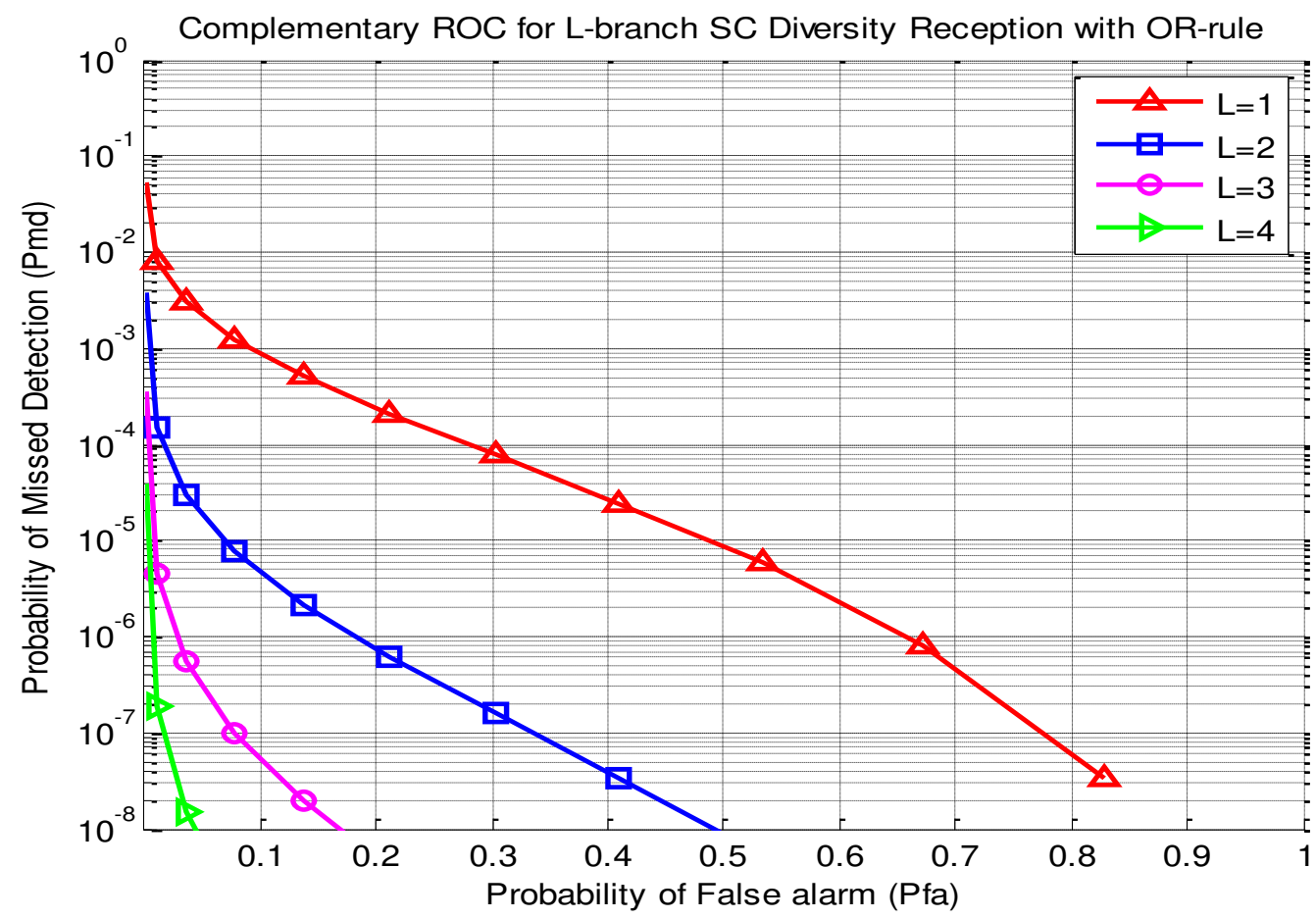

Fig 1(b): Complementary ROC curves for $\mathrm{L}$-branch $\mathrm{SC}$ reception $(\mathrm{u}=5, \mathrm{~N}=4, \mathrm{SNR}=20, \mathrm{~L}=1,2,3,4)$. 


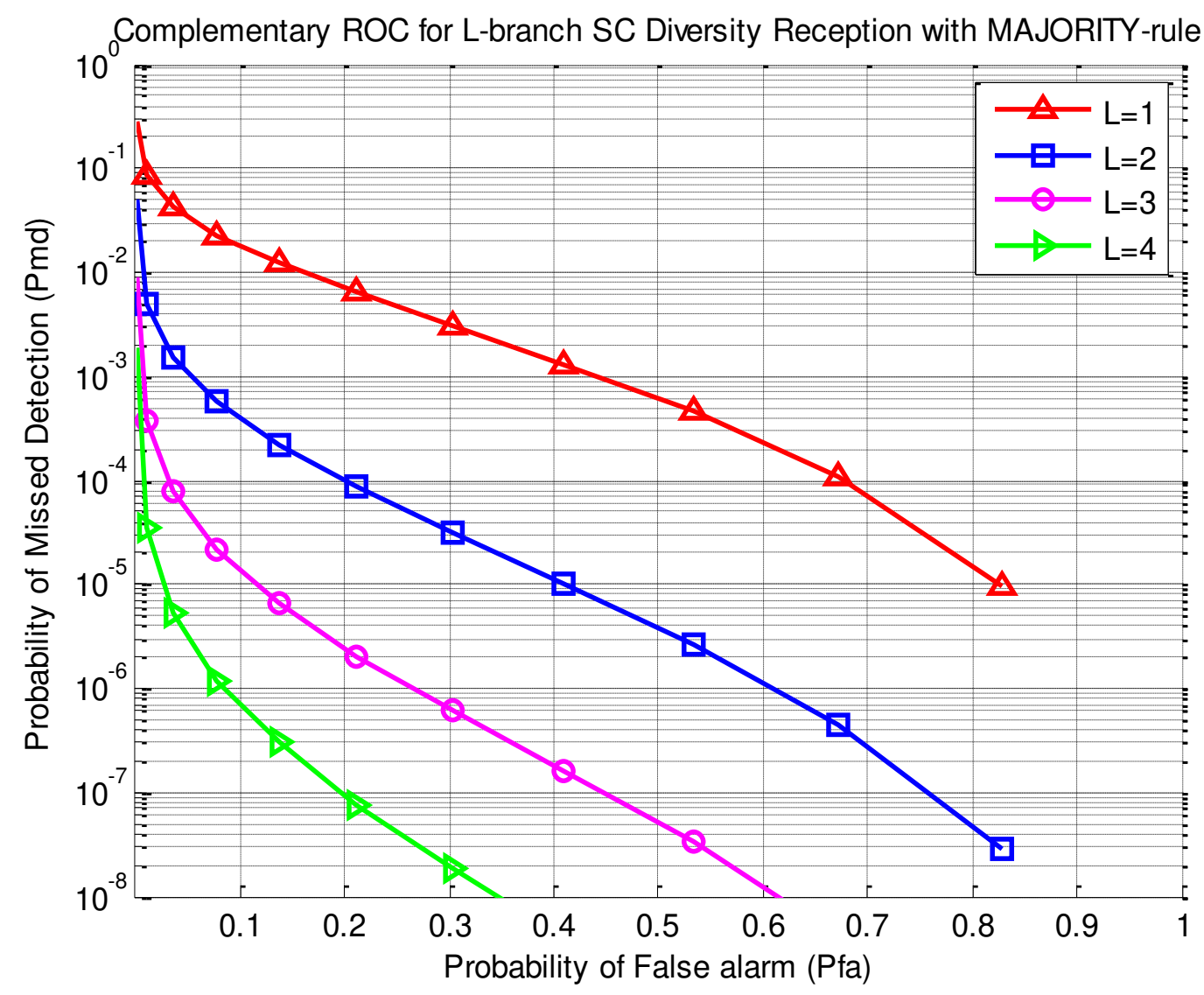

Fig 1(c): Complementary ROC curves for L-branch SC reception $(\mathrm{u}=5, \mathrm{~N}=4, \mathrm{SNR}=20, \mathrm{~L}=1,2,3,4)$.

Fig 1(a), Fig 1(b), and Fig 1(c) show complementary ROC curves of the 4 user's spectrum sensing in Rayleigh fading following AND rule, OR rule and MAJORITY rule respectively. It is also shown form the figure. Average SNR and $u$ are assumed to be $20 \mathrm{~dB}$ and 5 respectively. It is clearly depict from the Fig 1(a) the probability of false alarm increase under probability of missed detection with AND rule. From Fig 1(b) have shown that probability of false rate dramatically achievable decrease under probability of missed detection with OR rule. The number of individual users get their value of probability of false alarm. The figure clearly shows that suddenly change the probability of false depends on several users which is gives the desire results of OR rule. In the last Fig 1(c) have shown that comparatively probability of false alarm increase above two figures with MAJORITY rule. In the light of among three figures we can easily determine that the OR rule gives better performances than other rule.

\section{CONCLUSEION:}

We have studied hard decision based cooperative spectrum sensing over different fading channel in cognitive radio. Performance of cooperative spectrum sensing over Rayleigh, Ricean and Nakagami fading are presented and compared. It has been found that probability of missed detection is decreased by using different hard decision fusion rules. We observe that the OR rule has the better performance than AND, and MAJORITY rule in various channels. We also observe that spectrum sensing is harder in presence of Rayleigh and Nakagami fading and performance of energy detection degrades more in Nakagami channels than Rayleigh and Ricean channels. In Ricean channel, because of the LoS is signal, the sensing performance is better than in other channels. Furthermore, spectrum sensing in Ricean fading has better results than others.

\section{ACKNOWLEDGEMENT:}

Thank those who work on this topic of the research study to complete successfully.

\section{CONFLICTS OF INTEREST:}

The authors declared there are no conflicts of interest to publish the present research study. 


\section{REFERENCES}

1. Ahmed S, Alamgir M. H, Shamim M. H and Ibrahim M. A., (2012). "Cooperative Spectrum Sensing over Rayleigh Fading Channel in Cognitive Radio", IJECSE, 1(4), pp. 25832592.

2. Alamgir M. H, Shamim M. H, and Ibrahim M. A. (2012). "Cooperative Spectrum Sensing over Fading Channel in Cognitive Radio," International J. of Innovation and Applied Studies, 1(1), pp. 84-93.

3. Alamgir M. H, Ahmed S, Shamim M. H and Ibrahim M. A, "Performance of Cooperative Spectrum Sensing for Different Number of CR users in Cognitive Radio", International J. of Science and Research, 1(3), pp.145-149. https://www.ijsr.net/get abstract.php?paper id= IJSR12120365

4. Alam N., and Alam M. (2020). The trend of different parameters for designing integrated circuits from 1973 to 2019 and linked to Moore's law, Aust. J. Eng. Innov. Technol., 2(2), 16-23.

https://doi.org/10.34104/ajeit.020.016023

5. Chen Q., Gao F., Nallanathan A., and Xin Y. (2008). "Improved cooperative spectrum sensing in cognitive radio," In Proc. IEEE VTC 2008 Spring, , pp.1418-1422. https://doi.org/10.1109/VETECS.2008.298

6. Chen Q., Motani M., Wong W.-C., and Nallanathan A. (2011). "Cooperative spectrum sensing strategies for cognitive radio mesh networks," IEEE J-STSP, 5(1), pp.5667.

https://doi.org/10.1109/JSTSP.2010.2060468

7. Digham F. F., Alouini M. S., and Simon M. K. (2003). "On the energy detection of unkown signals over fading channels," in Proc. IEEE ICC, pp. 3575-3579.

8. Digham F. F., Alouini M. S., and Simon M. K. (2007). "On the energy detection of unknown signals over fading channels," IEEE Trans. Communications, 55(1), pp. 21-24. https://ieeexplore.ieee.org/document/4063501

9. FCC, (2002). "Spectrum Policy Task Force," 11/2002, ET Docket 02-135.

10. Ganesan G. and Li Y. G. (2007). "Cooperative spectrum sensing in cognitive radio-part I: two user networks," IEEE
Trans. Wireless Commun., 6(6), pp. 22042213. https://doi.org/10.1109/TWC.2007.05775

11. Ganesan G. and Li Y. G. (2007). "Cooperative spectrum sensing in cognitive radio part II: multiuser networks," IEEE Trans. Wireless Commun., 6(6), pp. 2214-2222. https://doi.org/10.1109/TWC.2007.05775

12. Gradshteyn I. S. and Ryzhik I. M. (2007). Table of Integrals, Series, and Products, $7^{\text {th }}$ ed. Academic Press.

13. Herath S., Rajatheva N., and Tellambura C., 2009 "Unified approach for energy detection of unknown deterministic signal in cognitive radio over fading channels," in Proc. IEEE ICC Workshops, pp.1-5. https://doi.org/10.1109/ICCW.2009.5208031

14. Herath S.P., Nandana Rajatheva, and Chintha Tellambura, (2009a) "On the energy detection of unknown deterministic signal over Nakagami channels with selection combining," in Proc. CCECE, pp. 745-749.

15. Islam S, Islam MS, and Mandal S. (2020). One dimensional heat transfer through a uniform plane wall by using finite volume method, Aust. J. Eng. Innov. Technol., 2(2), 24-30.

https://doi.org/10.34104/ajeit.020.024030

16. Lee Seung-Hwan, Oh Dong-Chan, and Lee Yong-Hwan, (2009). "Hard Decision Combining based Cooperative Spectrum Sensing in Cognitive Radio Systems". IWCMC 2009, Leipzig, Germany, June 21-24. https://doi.org/10.1145/1582379.1582576

17. Mishra S. M., Sahai A., and Brodersen R. W. (2006). "Cooperative sensing among cognitive radios," in Proc. IEEE Int. Conf. on Commun. 4, pp.1658-1663. https://doi.org/10.1109/ICC.2006.254957

18. Nallagonda S., Roy S. D. and Kundu S. (2011). "Performance of cooperative spectrum sensing with censoring of cognitive Radios in Rayleigh Fading Channel", in Proc. of IEEE INDICON 2011, December.

19. Nallagonda S., Roy S. D. and Kundu S., "Cooperative spectrum sensing with censoring of cognitive Radios in Rayleigh Fading Channel", in Proc. of IEEE Eighteenth National conference on Communications (NCC 2012), February. https://doi.org/10.1109/ANTS.2013.6802864 
20. Spyros Kyperountas, Neiyer Correal, Qicai Shi and Zhuan Ye, (2008). "Performance analysis of cooperative spectrum sensing in Suzuki fading channels," in Proc. of IEEE International Conference on Cognitive Radio Oriented Wire-less Networks and Communications, pp. 428-432. https://doi.org/10.1145/1582379.1582576

21. Stuber G. L. (2002). Principles of Mobile Communications, $2^{\text {nd }}$ ed. Norwell, MA: Kluwer Academic Publishers.

22. Sun H., Laurenson D., and Wang C.X. (2010). "Computationally tractable model of energy detection performance over slow fading channels," IEEE Comm. Letters, 14(10), pp. 924-926.

https://doi.org/10.1109/LCOMM.2010.090710. $\underline{100934}$
23. Urkowitz H. (1967). "Energy detection of unknown deterministic signals," in IEEE Proceedings, 55(4), pp. 523-531.

https://ieeexplore.ieee.org/document/1447503

24. Wang C.X., Chen H.H., Hong X., and. Guizani M. (2008). "Cognitive radio network manage-ment: tuning in to real-time conditions," IEEE Vehicular Technology Magazine, 3(1), pp.28-35.

25. Wang C.X., Hong X., Chen H.H., and. Thompson J. S. (2009). "On capacity of cognitive radio networks with average interference power constraints," IEEE Trans. Wireless Comm., 8(4), pp.1620-1625. https://doi.org/10.1109/TWC.2009.071075

Citation: Khan MF., and Hossain MA. (2021). Cooperative spectrum sensing with selection diversity reception in cognitive radio, Int. J. Mat. Math. Sci., 3(1), 1-7.

https://doi.org/10.34104/ijmms.021.0107 